\title{
FDA resists regulatory role in gene tests
}

Washington. A government-sponsored task force is debating whether to recommend that the US Food and Drug Administration (FDA) take on a role in regulating genetic tests offered by specialized laboratories even though the FDA itself has indicated a strong reluctance to do so.

The joint National Institutes of Health/ Department of Energy Task Force on Genetic Testing may shortly decide to call for some degree of regulatory oversight of these genetic tests by the FDA, particularly those whose results need careful interpretation. This would be spelled out in draft recommendations that the task force plans to publish in January, before it makes final recommendations in March.

But the suggestion that the FDA should step into the controversial area is meeting resistance at the agency. FDA officials argue that they do not have the resources to regulate genetic tests, and that to do so would open the agency to having to take responsibility for regulating many other in-house, non-genetic tests currently offered by sophisticated 'reference' laboratories.

Neil Holtzman, director of genetics and public policy studies at the Johns Hopkins Medical Institutions, and chair of the task force, said last week that it is developing a classification scheme which ranks tests according to their level of "potential danger" to the public. It may ask the FDA to regulate those defined as most dangerous. Holtzman has expressed a personal belief that the growing field may not be manageable without some degree of regulation.

The task force is most concerned, says Holtzman, about tests in which a positive result does not imply a high probability that an individual will develop the disease in question; those in which a negative result does not imply a high probability that the individual will remain free from the disease; and those for mutations predisposing to diseases - such as Alzheimer's - for which there is no known or accepted treatment.

Holtzman's comments follow the commercial introduction late last month of full sequence testing of $B R C A 1$ and $B R C A 2$ genes by Myriad Genetics of Salt Lake City, Utah. Mutations in the two genes imply a strong predisposition to - but not the certainty of - breast and ovarian cancers in women with family histories of the disease.

The company is charging $\$ 2,400$ for initial testing, and $\$ 395$ for tests of additional family members. Testing is available only through doctors. The company has promised to provide full counselling on the implications of the results of its tests; but the move has rekindled the debate over whether the FDA should be given authority to judge whether such efforts are adequate.

Agency officials say this new responsibility would overtax their finite resources, forc- ing them to cut back other commitments. At a task force meeting in September, Steve Gutman, a director of the FDA's Division of Clinical Laboratory Devices, protested that asking the agency to regulate genetic tests would be opening "an incredible universe" which would require "a new branch" to be handled properly. "What should we stop doing so we can do genetic tests?" he asked.

The agency's top official in the area agrees. "Should we be putting our resources into automated pap-smear readers [that affect millions of women every year], or into genetic tests that are available through a limited number of reference laboratories?" asks Bruce Burlington, director of the FDA's Center for Devices and Radiological Health.

In theory, genetic tests already fall under FDA regulation. The US Food, Drug and Cosmetic Act gives the FDA broad control over all in-vitro diagnostic products, including — according to the agency — tests

\section{IMAGE UNAVAILABLE FOR COPYRIGHT REASONS}

Homing in: screening can reveal susceptibility to disease, but intepretation is uncertain.

administered as in-house services by specialized laboratories.

In practice, however, the agency has not regulated these tests, focusing instead on kits and reagents produced for sale, for example, to other labs and doctors' offices. No such kits are believed to exist currently for genetic tests. The FDA regulates the gamut of other kits, from home pregnancy test kits to cell counters used by hospitals and laboratories for standard blood tests.

The distinction between the FDA's treatment of kits and in-house tests has not been lost on companies such as Myriad, which has made $B R C A 1$ and $B R C A 2$ tests available only as in-house services Similarly, Genzyme, of Cambridge, Massachusetts, offers in-house genetic testing for cystic fibrosis, and OncorMed, of Gaithersburg, Maryland, also tests in-house for mutations in $B R C A 1$ and $B R C A 2$ genes.

Under the Clinical Laboratory Improvement Amendments (CLIA) of 1988, companies offering in-house laboratory tests as services - from the production of monoclonal antibodies that detect rare diseases to routine microbiological cultures - are monitored not by the FDA but by the Health
Care Financing Administration and the Centers for Disease Control and Prevention.

CLIA requires merely that in-house tests be 'analytically' valid, meaning that they accurately measure what they are meant to measure - in the case of genetic tests, that a given mutation is present. But, in contrast to the FDA's regulatory requirements, there is no standard under CLIA for 'clinical validity', which in the case of genetic tests would essentially boil down to the ability of a test to predict future disease.

According to Holtzman, the lack of such a requirement under CLIA has left the task force with "tremendous doubts" about the capability of the act to assure the quality of genetic tests.

The FDA disagrees. It has proposed a rule that acknowledges its regulatory authority over reagents used in in-house tests by specialized laboratories. But it has also said that it does not plan to regulate these reagents - including gene probes used in genetic tests - implying that such oversight would be left to CLIA.

The FDA has collected public comment on its proposed rule, and plans to offer a final version soon. Meanwhile, said Holtzman, companies marketing genetic tests as in-house services "say 'trust us". So far, he adds, such companies have not shown themselves untrustworthy. "The problem is, can we be sure that other companies will be as responsible?"

Patricia Barr, an attorney who works with the National Breast Cancer Coalition and is a member of the task force, says that her group is "terribly concerned about clinical validation". It would like to see FDA approval tied to guarantees by companies that they will gather long-term data on patients who receive their tests.

But the companies are fighting to preserve the status quo. Patricia Murphy, vice-president of genetic services at OncorMed, told Congress this summer that the FDA is "not the appropriate agency to regulate genetic tests" conducted in reference laboratories. Myriad has warned that any FDA role "could stifle innovation and diffusion of genetic testing technologies".

Janet Haskell, president of Myriad Genetic Laboratories, the company's genetic testing facility, argues that, in the laboratory setting, "there's really no difference" between a blood cholesterol test and a genetic test. "No-one has given me a good reason why this test, or that type of test, would require FDA oversight, whereas others do not," she says.

The 15-member task force is expected to decide its position at a meeting early next month. Its eventual recommendations will be published for comment before being agreed in their final form next March.

Meredith Wadman 